Supplementary Information for the article:

\title{
Hydrothermal liquefaction of softwood: selective chemical production under oxidative conditions
}

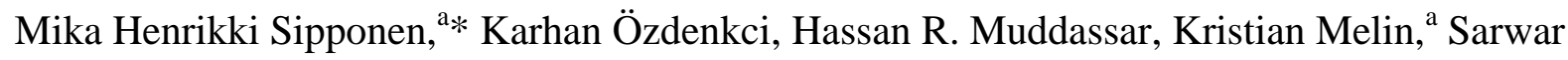
Golam, Pekka Oinas

Aalto University School of Chemical Technology, Department of Biotechnology and Chemical Technology, Espoo, Finland.

a: Present address: VTT Technical Research Centre of Finland Ltd, Espoo, Finland

*Corresponding author: Tietotie 2, Espoo, P.O. Box 1000, FI-02044 VTT, Finland (postal address);

+358401242757 (phone); mika.sipponen@vtt.fi (email) 


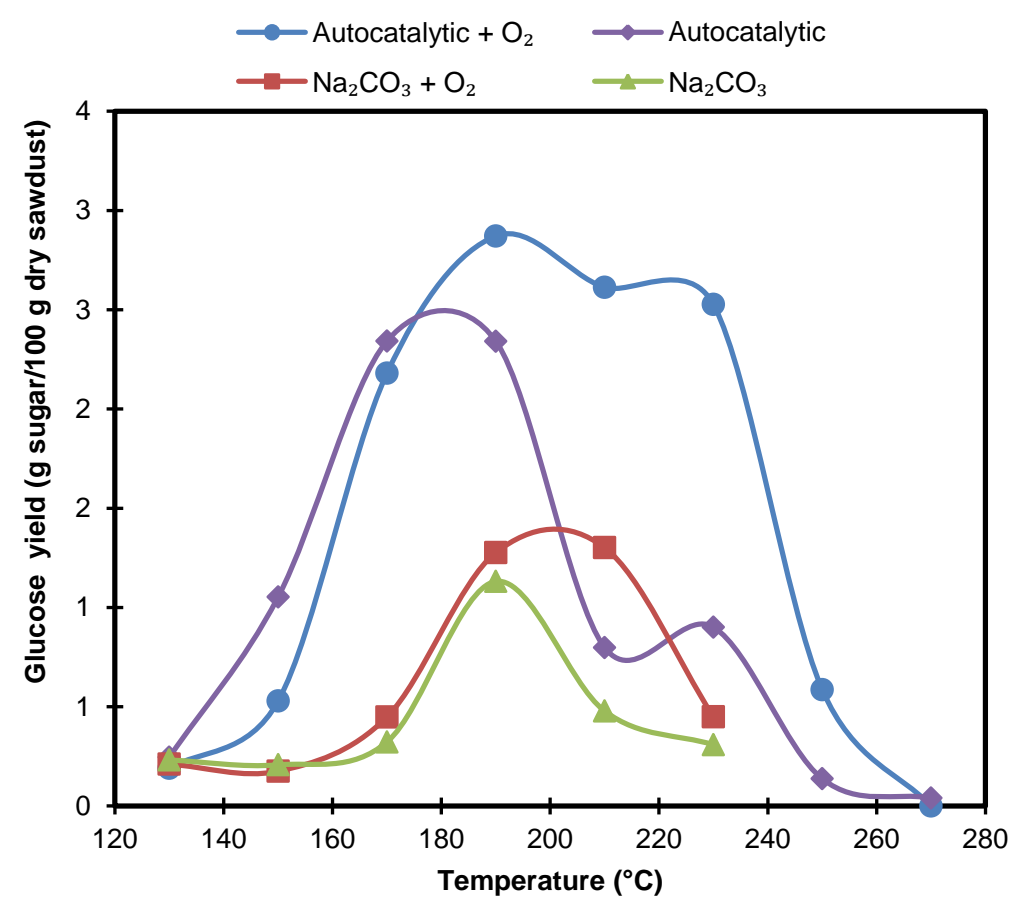

Figure S1. Glucose yields (g from $100 \mathrm{~g}$ dry wood) in hydrothermal liquefaction of softwood. Autocatalytic and sodium carbonate containing liquefaction was carried out as a function of temperature and in the presence or absence of molecular oxygen. 

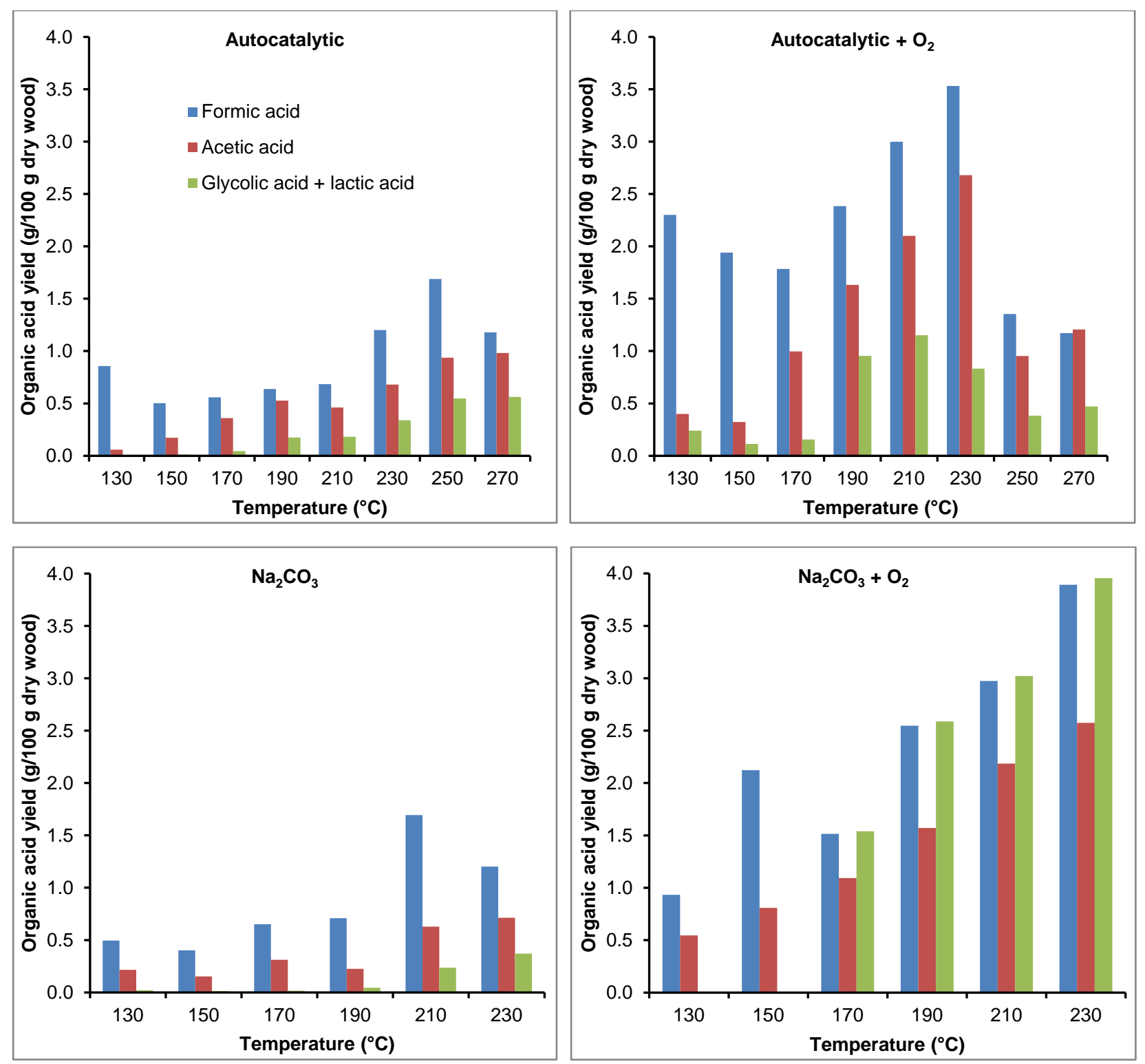

Figure S2. Organic acid yields ( $\mathrm{g}$ from $100 \mathrm{~g}$ dry wood) in hydrothermal liquefaction of softwood. Autocatalytic and sodium carbonate containing liquefaction was carried out as a function of temperature and in the presence or absence of molecular oxygen. Colour coding of the bars is similar in each figure. 


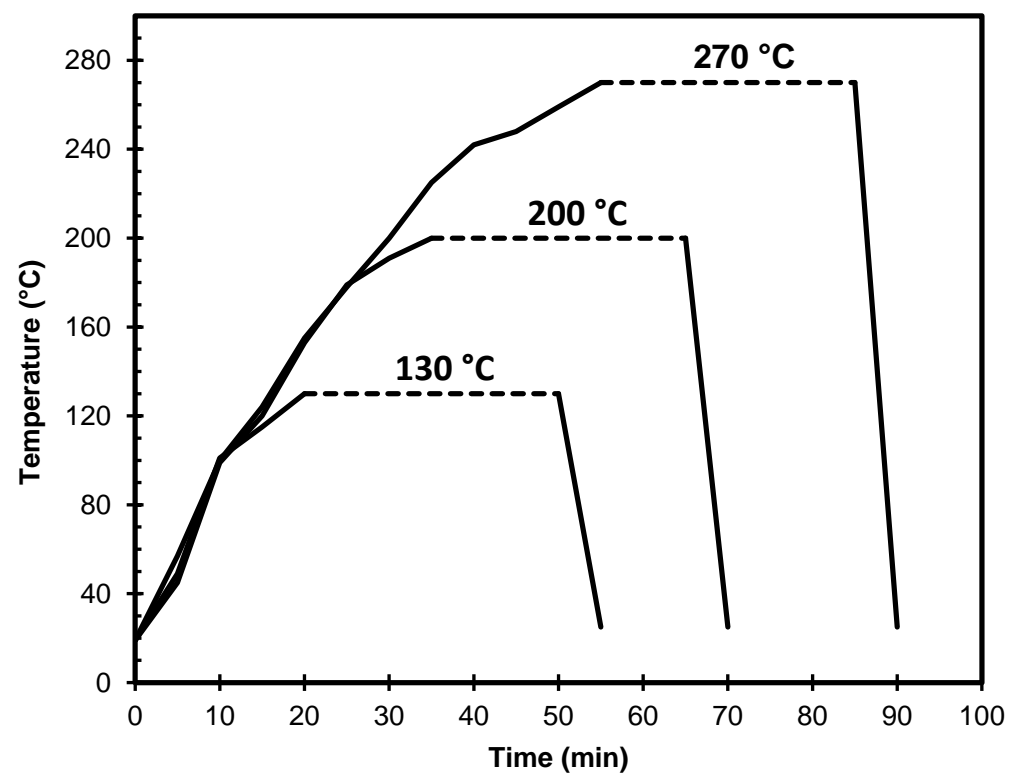

Figure S3. Illustrative examples of the heating, isothermal (dashed lines) and cooling stages of the pressure reactor used in the HTL experiments. 